\title{
Bibliographie de Michèle Therrien
}

Professeure des Universités émérite, Langue et culture inuit, CERLOM, Inalco

\section{Marie Mauzé}

\section{(2) OpenEdition}

\section{Journals}

Édition électronique

URL : https://journals.openedition.org/jsa/17038

DOI : 10.4000/jsa. 17038

ISSN : 1957-7842

Éditeur

Société des américanistes

\section{Édition imprimée}

Date de publication : 15 juin 2019

Pagination : 197-205

ISBN : 978-2-902715-11-4

ISSN : 0037-9174

Référence électronique

Marie Mauzé, "Bibliographie de Michèle Therrien », Journal de la Société des américanistes [En ligne],

105-1 | 2019, mis en ligne le 15 juin 2019, consulté le 04 septembre 2022. URL : http://

journals.openedition.org/jsa/17038; DOI : https://doi.org/10.4000/jsa. 17038 


\title{
Bibliographie de Michèle Therrien
}

\author{
Professeure des Universités émérite, \\ Langue et culture inuit, CERLOM, Inalco
}

\author{
établie par Marie MAUZÉ*
}

\section{Ouvrages}

1979 La conférence tripartite fédérale, provinciale et inuit, Nouveau-Québec 1970, analyse socio-politique, EHESS/Centre d'études arctiques (Separata, 1), Paris.

1987 Le corps inuit. Québec arctique, Société anthropologique et linguistique de France (SELAF)/Presses universitaires de Bordeaux (Arctique, 1), Paris.

1999 Printemps inuit, naissance du Nunavut, préface de John Amagoalik (Jaan Amarualik), Indigène éditions, Montpellier; Prix Samuel de Champlain, Institut France-Amériques.

2012 Les Inuit, Les Belles lettres (Guide Belles lettres des Civilisations), Paris.

\section{Direction d'ouvrages}

1981 L'Arctique, La Documentation française (Problèmes politiques et sociaux, 412), Paris, $40 \mathrm{p}$.

1995 Peuples des Grands Nords, traditions et transitions, actes du colloque international [Unesco, 1993], textes recueillis par Anne-Victoire Charrin, Jean-Michel Lacroix et Michèle Therrien, Presses de la Sorbonne nouvelle/Institut national des langues et civilisations orientales, Paris.

1996 La dynamique dans la langue et la culture inuit, Nicole Tersis et Michèle Therrien (dir.), Peeters (Selaf, 361; Arctique, 4), Paris.

1999 Pour mieux comprendre le Nunavut, Sylvie Teveny et Michèle Therrien (dir.), actes [Journée Nunavut, en collaboration avec la formation continue de l'Inalco, 30 janvier 1999], Institut national des langues et civilisations orientales, avec le concours de l'Ambassade du Canada en France, Paris.

2000 Les langues eskaléoutes, Sibérie, Alaska, Canada, Groenland, Nicole Tersis et Michèle Therrien (dir.), collaboration de Charlotte Levanthal, CNRS Éditions (Sciences du langage), Paris.

* Laboratoire d'anthropologie sociale, Collège de France, EHESS, CNRS PSL Université de recherche [marie.mauze@college-de-france.fr]. 
2001 avec Frédéric Laugrand (dir.), Interviewing Inuit elders (Ilisapi Ootoova, Tipuula Qappik Atagutsiak, Tirisi Ijjangiaq, Jaikku Pitseolak, Aalasi Joamie, Akisu Joamie, Malaija Papatsie), Vol. 5. Perspectives on traditional health, Language and Culture Program of Nunavut Arctic College, Iqaluit; version inuktitut, sous le titre Aanniarniq aanniaqtailimaniq.

2003 Les Inuit de l'Arctique canadien, Michèle Therrien (dir.), textes recueillis par Pauline Huret, préface Michèle Therrien, CIDEF/Association internationale francophone/Inuksuk/Langues O' (Francophonies), Québec.

2004 Dynamics and shifting perspectives Arctic societies and research. Proceedings of the First IPSSAS Seminar, Nuuk 2002, Michèle Therrien (dir.), Ilisimatusarfik University of Greenland, Nuuk.

2007 Entretiens avec des ainés inuit (Ilisapi Ootoova, Tipuula Qappik Atagutsiak, Tirisi Ijjangiaq, Jaikku Pitseolak, Aalasi Joamie, Akisu Joamie, Malaija Papatsie), Michèle Therrien et Frédéric Laugrand (ed.), Vol. 5. Bien-être physique et psychique, Programme « Langue et culture » du Nunavut Arctic College, Iqaluit, http://www.traditional-knowledge.ca/, consulté le 07/06/2019.

2008 Paroles interdites, Michèle Therrien (dir.), Karthala/Langues O', Paris.

2009 Proceedings of the 15th Inuit Studies Conference, Orality in the 21st century. Inuit discourse and practices, Béatrice Collignon et Michèle Therrien (dir.), Inalco, Paris, CD-ROM, http://www.inuitoralityconference.com, consulté le $07 / 06 / 2019$.

\section{Articles}

1974 «Études sur l'administration au Nouveau-Québec esquimau depuis 1969 », Inter-Nord, 13-14, p. 363-369.

1975 «Les Gens de Payne », Le Courrier de l’Unesco, janvier, p. 21-26.

1976 «L'avenir des Inuit du Nouveau-Québec après la signature du premier traité bipartite devant ouvrir les régions nordiques au développement accéléré », Actes du XVII Congrès international des américanistes, Congrès du Centenaire, Paris, vol. V, p. 155-175.

1978a «La Convention de la baie James et du Nord québécois », Inter-Nord, 15, p. 254-263.

1978 « « Sous le signe du pétrole, valeurs en péril », Vivant Univers, 314 (janv.-fév.), p. 2-22.

1979 « An ethnological regard on a linguistic phenomenon », in Eskimo languages, their present-day conditions, University of Aarhus and Arkona Publishers, Aarhus, p. 155-159.

1982a « La Maison de neige (illuvigaq), une métaphore du corps humain », InterNord, 16, p. 121-126.

1982b « Le fonds de la bibliothèque du Centre d'études arctiques », Arctica, CNRS Éditions (Colloques du CNRS), p. 103-109.

1984 «Les Inuit face à l'équilibre menacé », in Mer, l'autre territoire, le Québec face à la mer, 10 (juin-juillet), p. 48-51.

1985a «L'entreprise Revillon Frères. Réactions euro-canadiennes, amérindiennes et inuit au commerce des fourrures », in Xavier Pons et Marcienne Rocard (dir.), 
Colonisations. Rencontres Australie-Canada, [colloque, 15-17 mars 1984, Toulouse], Université de Toulouse-Le Mirail (Travaux de l'université de Toulouse-le-Mirail. Série B, 7), Toulouse, p. 19-26.

1985 b en collaboration avec Raymonde Litalien, «Archives Revillon. La traite des fourrures au $\mathrm{Xx}^{\mathrm{e}}$ siècle », L'archiviste [Archives nationales du Canada, Ottawa], 12 (3 [mai-juin]), p. 16-18.

1985c «L'Inuk tel que le révèle le langage sacré », Inter-Nord, 17, p. 111-114.

1985d «Portfolio commenté: Robert J. Flaherty », L'Autre Journal, Nouvelles Littéraires, 2 (janvier), p. 28-36.

1986a «Représentations et pratiques liées à l'expérience de l'habitat chez les Inuit de l'Arctique oriental canadien. Approche ethnolinguistique », Ethnologie et anthropogéographie arctiques, CNRS, Paris, p. 209-215.

1986 b «elations inter-ethniques et commerce des fourrures, les carnets de voyage du capitaine Thierry Mallet, Revillon Frères, 1920-1925», Recherches amérindiennes au Québec, 26 (4), p. 35-46.

1987 «La Parole partagée, l'homme et l'animal arctiques », Cahiers de littérature orale, 22, p. 105-130.

1989 «Identité et forme graphique, le syllabaire des Inuit de l'Arctique oriental canadien ", L'Ethnographie, 105, 85 (1), p. 71-88.

1990 «Traces sur la neige, signes sur le papier. Significations de l'empreinte chez les Inuit Nunavimmiut », Journal de la Société des américanistes, 76, p. 33-53.

1991a «La chasse et l'imaginaire inuit, ce que la langue inuit révèle du kayak (qajaq) et de la chasse (Arctique oriental canadien)», Arctique Horizon 2000. Les peuples chasseurs et éleveurs, CNRS Éditions, Paris, p. 149-165.

1991b « Les Inuit », in Pierre Bonte et Michel Izard (dir.), Dictionnaire de l'Ethnologie et de l'Anthropologie, PUF, Paris, p. 386-388.

1991c «Knud Rasmussen », in Pierre Bonte et Michel Izard (dir.), Dictionnaire de l'Ethnologie et de l'Anthropologie, PUF, Paris, p. 615-616.

1991d «La Langue inuit, une langue foisonnante », La traduction littéraire scientifique et technique, La Tilv (Paroles et actes), Paris, p. 70-84.

1992a « Inuit/Esquimaux (Sibérie, Alaska, Canada, Groenland) », in Jean Poirier et Jean François Mattei (dir.), L'Encyclopédie philosophique, vol. 3. Les œuvres philosophiques, t. 2. L'Amérique du Nord et centrale, études ethnologiques $X I X-X X^{e}$ siècles, PUF, Paris, p. 4219-4223.

1992b «L'identité bien construite des Inuit de l'Arctique canadien », in Jean Michel Lacroix et Fluvio Caccia (dir.), Métamorphoses d'une utopie, textes recueillis par Le pluralisme ethno-culturel en Amérique: un modèle pour l'Europe, Sorbonne, Paris, p. 193-203.

1992c «Revillon Frères et la traite des fourrures au Canada au début du Xx siècle », in La traite de la fourrure, les Français et la découverte de l'Amérique du Nord, Musée du Nouveau Monde, La Rochelle/Éditions de l'Albaron, Thononles-Bains, p. 106-137.

1993a «Membres de la direction Revillon Frères et chefs de poste: deux points de vue sur la traite de la fourrure », Études inuit/Inuit studies, 17 (2), p. 49-63. 
1993 « « Le syllabaire des Inuit ou l'attachement à la pratique identitaire dans l'Arctique oriental canadien », in Marie-Josée Jolivet et Diana Rey-Hulman (dir.), Jeux d'identités. Études comparatives à partir de la Caraïbe, L'Harmattan, Paris, p. 83-100.

1995a «Corps sain, corps malade chez les Inuit, une tension entre l'intérieur et l'extérieur, entretiens avec Taamusi Qumaq ", Recherches amérindiennes au Québec, 20 (1), p. 71-84.

1995 b en collaboration avec Bruno Vercier, «Préface », in Yves Thériault, Agoak. L'héritage d'Agaguk [réédition], Éditions de l'Archipel, Paris, p. 7-14.

1995c « Les traditions culinaires des Inuit », in Michel Aufray et Michel Perret (dir.), Cuisines d'Orient et d'ailleurs, traditions culinaires des peuples du monde, Glénat, Grenoble, p. 318-321.

1995d " "Inuktitut", notice biographique », in Pierre Labrousse (dir.), Langues $O$ ' 1795-1995. Deux siècles d'histoire de l'École des langues orientales, Editions Hervas, Paris, p. 382-383.

1995e «Tradition et transition, la notion de dynamique chez les Inuit», in Peuples des Grands Nords traditions et transitions, textes recueillis par Anne-Victoire Charrin, Jean-Michel Lacroix et Michèle Therrien, Presses de la Sorbonne nouvelle/Institut national des langues et civilisations orientales, Paris, p. 245-254.

1995 « «nuktitut», in Bicentenaire des Langues O'. Le tour du monde en 80 langues, texte intégré au spectacle d'Alain Germain, L'Asiathèque, Paris p. 86-87.

1996a «Les expériences premières et la notion de dynamique généralisée », in Nicole Tersis et Michèle Therrien (dir.), La dynamique dans la langue et la culture inuit, Peeters (Selaf, 361; Arctique 4), Paris, p. 22-41.

1996 b « Néologie inuit et nouvelles traductions de la culture », Études canadiennes/ Canadian Studies, 41, p. 25-39.

1997a «Inuit concepts and notions regarding the Canadian Justice System », in Desmond Brice-Bennett (dir.), Maligaliriniup uqausiqarvingat. Inuktitu, qallunaatitut, uivititullu. Legal glossary (English, Inuktitut, French)/Glossaire juridique (anglais, inuktitut, français), Ukiuqtaqtumi silaktusarvik, Nunatta ilisarvingat $=$ Arctic College, Nunatta Campus $=$ Collège de l'Arctique, Iqaluit, p. 250-275 [1st ed. 1996].

1997b « Mythes et contes inuit », La Chaussure, revue de l'Institut de calcéologie, 5, p. 46-53.

1998a «Comme un insecte surpris par le froid », Pratiques, les cahiers de la médecine utopique, 2, Indigène éditions, Montpellier, p. 80-81.

1998 b «Grand Nord canadien, les Inuit et la transmission du savoir », Diagonales, 47, p. 38-39.

1998c «Printemps inuit au Nunavut», La lettre des Langues O’, 5 (décembre), p. 7-8.

1998d «Le baiser et la chance », in Diana Rey-Hulman et Michel Boccara (dir.), Odeurs du monde. Écriture de la nuit, L'Harmattan/Inalco, Paris, p. 203-208.

1998e «Les exigences du célèbre rire inuit », in Éliane Daphy et Diana ReyHulman (dir.), Paroles à rire, Inalco (Colloques Langues O'), Paris, p. 211-222.

1999 "All Qallunaat predicted our extinction: some Inuit points of views on identity", in Jarich Oosten et Cornelius Remie (dir.), Arctic identities, continuity 
and change in Inuit and Saami Societies, Research School CNWS, Leiden University, Leiden, p. 28-35.

2000a en collaboration avec Nicole Tersis, « Adéquation de la traduction dans une langue de type modulaire: la langue inuit», in Thomas Szendé (dir.), Dictionnaires bilingues méthodes et contenus, Honoré Champion, Paris, p. 15-23.

2000b «CANADA. Création d'un nouveau territoire, le Nunavut », Universalia 2000, Encyclopédie Universalis, Paris, p. 144-148.

2000c «Paroles inuit. Alaska, Arctique canadien, Groenland», avec la collaboration de Dominique Bulle et Vladimir Randa, in Stanislas Rodanski, Jaime Sabine, James Brook (dir.), Paroles inuit (n ${ }^{\circ}$ spécial de Poésie 2000, 82/avril), Maison de la Poésie, Paris, p. 8-51.

2000d «Nouvelles terminologies en inuktitut, contraintes logiques, linguistiques et culturelles ", in Nicole Tersis et Michèle Therrien (dir.), Les langues eskaléoutes, Sibérie, Alaska, Canada, Alaska, CNRS Éditions (Sciences du langage), Paris, p. 283-301.

2000e «Les Inuit et le choix de la modernité, l'arme de la néologie », in Miura Nobutaka et Kasuya Keisuke, Les impérialismes linguistiques hier et aujourd'hui, à l'Est et à l'Ouest [en japonais], Fujiwara-shoten Publishing Company, (collaboration Maison franco-japonaise à Tokyo/université de Hitotsubashi/Inalco), Tokyo, p. 238-246.

2001a «Un territoire placé sous la responsabilité inuit: le Nunavut. Pérennité des valeurs dans un monde changeant ", in Pascal Lièvre (dir.), Logistique en milieux extrêmes, principes d'organisation des expéditions polaires à ski, Hermès Science Publications, Paris, p. 101-111.

2001b «Enseignements et recherches en France concernant les Premières nations et les Inuit », in Jean-Michel Lacroix (dir.), États des lieux de la recherche sur le Canada en France (1976-2001), Association française d'Études canadiennes, Bordeaux, p. 177-209.

2001c «La pérennité des valeurs inuit: l'exemple du Nunavut », in Marie-Françoise Labouz Intégrations et identités nord-américaines vue de Montréal 1995-2000, Bruylant, Bruxelles, p. 39-50.

2001d «Le Nunavut, un lieu de réflexion et peut-être un modèle transposable », Peuples premiers. Cultures traditionnelles et modernité dans l'espace francophone, séminaire du Haut Conseil de la Francophonie 6-7 décembre 2000, p. 12-20.

2001e «La question du concept Inuit qaujimajatuqangit et le bilan du jeune gouvernement du Nunavut », Études canadiennes/Canadian Studies, 52, p. 131-147.

2002a «Rêves d'une apprentie-chamane inuit», Cahiers de littérature orale, 51 (nº spécial « Récits de rêves », Nicole Belmont [dir.]), p. 169-183.

2002b «Un monde de liens et d'itinéraires», in Michel Côté et al. (dir.), Inuit. Quand la parole prend forme, catalogue de l'exposition [Musée d'histoire naturelle de Lyon, futur Musée des cultures du monde, 17 déc. 2002-18 mai 2003], Éd. Glénat, Grenoble, p. 16-21.

2002c «Arctique inuit », in Eve Sivadjian (dir.), GEO Déserts du monde, Solar, Paris, p. 182-191. 
2002d «Ce que précise la langue inuit au sujet de la remémoration », Anthropologie et Sociétés, 26 (2-3), p. 117-135.

2003 «Quelques lieux de la réflexion et de l'action inuit», Réseaux autochtones, partenariats, questions d'éthiques, Muséum d'histoire naturelle de Lyon, Lyon, p. 153-159.

2004a «How semantic information can contribute to shed light on concepts we want to investigate », in Michèle Therrien (dir.), Dynamics and shifting perspectives Arctic societies and research. Proceedings of the First IPSSAS Seminar, Nuuk 2002, Ilisimatusarfik University of Greenland, Nuuk, p. 241-252.

2004b «Le système de numération des Inuit de l'Arctique canadien », in BrunoPerrone et Thierry-Petitclerc (coord.), VII Symposium mathématiques et dialyse, Méditor, Strasbourg, p. 99-105.

2005 «Corps inuit, espace géographique et cosmologique », in Marie-Françoise André, Le monde polaire: mutations et transitions, Ellipses (Carrefours), Paris, p. 39-52.

2006a «Les Inuit et le choix de la modernité: l'arme de la néologie », in Louis-Jean Calvet et Pascal Griolet (dir.), Impérialismes linguistiques hier et aujourd'hui, Inalco/Édisud, Paris, p. 233-239 (paru initialement en japonais).

2006 b «Résister à l'essentialisme. Cinq années d'exercice du pouvoir au Nunavut (Arctique canadien) », in Christian Gros et Marie-Claude Strigler, Etre Indien dans les Amériques, spoliation et résistance, mobilisations ethniques et politique du multiculturalisme, Éditions de l'Institut des Amériques, Paris, p. 295-303.

2007a «Un art contemporain », Vacarme, 39, p. 18-19.

2007 « «es Inuits confrontés aux risques du réchauffement des eaux arctiques », Le Monde, jeudi 19 juillet 2007 (Débats « l'Homme dans les conditions extrêmes »), p. 21.

2007c «Démocratie et reconnaissance: construire des partenariats de recherche », in Sérgio Paulo Rouanet et al. (dir.), Réinventer la démocratie? Diversité culturelle et cohésion sociale, PUF (Diogène: revue internationale des sciences humaines, 220), Paris, p. 133-136.

2008a « Nous empêcher de chanter et de danser, autant essayer de couper la langue d'un oiseau/To try to prevent us from singing and dancing is as futile as trying to cut out a bird's tongue », Totems et chamanes, arts anciens d'Alaska et de Colombie britannique, Galerie Flak, Paris, p. 53-83.

2008b « Avant-propos », in Michèle Therrien (dir.), Paroles interdites, Karthala/ Langues O', Paris, p. 9-27.

2008c «Tout révéler et rester discret chez les Inuit de l'Arctique canadien », in Michèle Therrien (dir.), Paroles interdites, Karthala/Langues O', Paris, p. 251-285.

2009 «The Inuit are expecting partnerships. We must hear this call for solidarity based on skill sharing/Les Inuit sollicitent des partenariats. Nous devons entendre cet appel fondé sur le partage des compétences », in Leslie Jessop (dir.), The call of the Poles. 100 prominent people worldwide commit to saving the poles/L 'appel des pôles. 100 personnalités du monde entier pour sauver les pôles, Le Cercle polaire, Paris, p. 242-243, http://www.lecerclepolaire.com/ fr/nos-actions/lappel-des-poles/l-appel-des-poles-2009 (consulté le 17/06/19). 
2010a avec Frédéric Laugrand, « Chez les Inuit Grand Nord canadien, handicap et performativité », in Charles Gardou (dir.), Le handicap au risque des cultures. Variations anthropologiques, Éditions Érès (Connaissances de la diversité), Toulouse, p. 75-94.

2010b avec Jacques Galinier, Aurore Monod-Becquelin, Guy Bordin, Laurent Fontaine, Francine Fourmaux, Juliette Roullet Ponce, Piero Salzarulo, Philippe Simonnot et Iole Zilli, « Anthropology of the night: cross-disciplinary investigations », Current Anthropology, 51 (6), p. 819-847.

2011a avec Nicole Tersis, « La famille eskaléoute », in Emilio Bonvini, Joëlle Busutill, Alain Peyraube (dir.), Dictionnaire des langues, PUF (Quadrige), Paris, p. 1339-1346.

2011b « Comment les Inuits voient-ils le monde? », in Fabienne Lemarchand, MarieFrançoise André et Frédérique Rémy (dir.), Cap sur les pôles, 100 questions sur les mondes polaires, Omniscience, Montreuil, p. 166-167.

2011c «Quel avenir pour les Inuits? », in Fabienne Lemarchand, Marie-Françoise André et Frédérique Rémy (dir.), Cap sur les pôles, 100 questions sur les mondes polaires, Omniscience, Montreuil, p. 196-197.

2012a «The Iqaluit/Paris/Iqaluit annual student exchange », in Louis-Jacques Dorais et Frédéric Laugrand (dir.), Linguistic and cultural encounters in the Arctic. Essays in memory of Susan Sammons, Centre interuniversitaire d'études et de recherches autochtones, Université Laval (Cahiers du CIÉRA, Hors-série), Québec, p. 15-18.

2012b « La question du genre intéresse-t-elle les Inuit? », in Féminismes, ailleurs, Indigènes éditions, Montpellier, p. 15-21.

2012c «"La parole fait de nous ce que nous sommes." Partage des savoirs chez les Inuit », in Mélanges offerts à Anne-Victoire Charrin, t. 2. De l'Asie russe et d'ailleurs ( ${ }^{\circ}$ spécial de Slovo, 39-40), Publications Langues'O, Paris, p. 235-256.

2012d «Comment les Inuits apprivoisent le climat », in Les pôles enjeu planétaire, Sophia publications (Les dossiers de la recherche, hors-série, 51), Paris, p. 52-54.

2013 «Les Inuit et le cybermonde », in Bernard Andrieu et Gilles Boetsch (dir.), Les corps du monde, atlas des cultures corporelles, Armand Colin, Paris, p. 36-39.

2014 «Les Inuit et la question du changement climatique », Orient, p. 87-93.

2015 «Vivants, défunts, animaux et esprits: échanges parlés chez les Inuit », in Michèle Cros, Julien Bondaz et Frédéric Laugrand (dir.), Bêtes à pensées. Visions des mondes animaux, Éditions des archives contemporaines, Paris, p. 165-188.

\section{Mémoire, thèse, dossiers, rapports}

1980 Lexique du corps et champs métaphoriques (dialecte du Nouveau-Québec), thèse de doctorat de $3^{\mathrm{e}}$ cycle, Centre d'études arctiques CNRS-EHESS.

1984 Archives Revillon photos. Inventaire analytique, Archives publiques du Canada à Paris, Paris, 220 p. (Ouvrage multigraphié et microfilm déposé aux Archives nationales à Ottawa.)

1990 « Les Inuit et le système judiciaire », dossier remis à Pierre Rousseau, procureur et avocat général, ministère de la Justice du Canada, bureau régional de 
Yellowknife et à Jean Auger, coordonnateur de la justice en milieu autochtone, ministère de la Justice du Québec, 32 p.

1992 Corps sain et corps malade chez les Inuit. Façons de vivre et de dire son corps. Entretiens avec Taamusi Qumaq, rapport rendu au département d'Anthropologie, université Laval et au FCAR (Fonds de recherche du Québec), février 1992, 90 p.

1996 Document de synthèse en vue de l'habilitation à diriger des recherches, Inalco, déposé en février, $98 \mathrm{p}$.

1996a Piqujaliriniq. Les Inuit et le système judiciaire, approche ethnosémantique, analyse de 200 termes inuit, dossier déposé au ministère de la Justice du Québec (mission Inalco), collaboration avec le département de Sociologie de l'université de Franche-Comté, 89 p.

2014 co-rédactrice de la section « Les sociétés autochtones et le changement global », in Prospective $d u$ " Chantier arctique français 2015-2020 », rapport remis aux autorités du CNRS, CNRS, Paris, p. 45-49, http://www.chantier-arctique. fr/fr/index.php (consulté le 17/06/19).

\section{Traductions, transcriptions}

2000 Kakoot. Récits du pays des caribous, texte présenté et annoté par Michèle Therrien, postface ethnohistorique de Yvon Csonka, Septentrion, Québec/ Sillery; traduction de l'anglais de Glimpses of the Barren Lands de Thierry Mallet (1930, 142 p.).

2007 Léger comme un flocon, poème en inuktitut, (Anonyme), images de Judith Gueyfier, traduit de l'inuktitut par M. Therrien, Rue du Monde (Petits Géants du monde), Paris.

2014 Macdonald Mary Ellen, Laakkuluk Williamson Bathory, Hannah Shenker, Hope Weiler, Margaret Berry, Atul Sharma et Rodd Celia, « Understanding healthy pregnancies: the perspective of Inuit midwives in northwestern Quebec ", avec la collaboration de Michèle Therrien pour la traduction et la validation des matériaux inuit, The Canadian Journal of Rural Medicine, 19 (4), p. 128-133.

\section{Matériel pédagogique}

1973 Programme de sciences humaines à l'élémentaire en milieu inuit, Commission scolaire du Nouveau-Québec, Québec, 400 p.; traduit en langue anglaise par Robert Frankling: Human Sciences for the Inuit at the Elementary Level, Nordic School Board, Québec, 1976, 230 p. (diffusé dans les écoles inuit par Kativik School Board, 5 chapitres, chacun étant dédié à une tranche d'âge).

1984 Timiup atingit, anatomie inuit, 450 termes (syllabaire et caractères latins), recherche déposée à l'Institut culturel inuit Avataq et à la Commission scolaire Kativik (mission CNRS 1983), ouvrage multigraphié utilisé dans les écoles inuit.

1989 Ilisautiksait, cours de langue inuit, Langues O', cahier d'exercices, multigraphié à l'intention des étudiants, 40 p., cahier $\mathrm{n}^{\circ} 1$. 
1991a Ilisautiksait, cours de langue inuit, Langues O', cahier d'exercices, multigraphié à l'intention des étudiants, 41 p., cahier $\mathrm{n}^{\circ} 2$.

1991b Ilisautiksait, cours de langue inuit, Langues O', cahier d'exercices, multigraphié à l'intention des étudiants, 37 p., cahier n ${ }^{\circ} 3$.

1993 Ilisautiksait, cours de langue inuit, Langues O', cahier d'exercices, multigraphié à l'intention des étudiants, 21 p., cahier n ${ }^{\circ} 4$.

1996 Ilisautiksait, cours de langue inuit, Langues O', cahier d'exercices, multigraphié à l'intention des étudiants, 39 p., cahier $\mathrm{n}^{\circ} 5$.

\section{Multimédia}

1998 Printemps inuit, upirngaksaangulirmat, réalisation M. Therrien, montage G. Sinelle et P. Hervouët, 60 minutes en collaboration avec le Cerpaim, Inalco, tourné à Iqaluit, capitale du Nunavut.

2001 Participation au Cédérom L'aventure des écritures, réalisation d'une fiche 10000 signes sur le syllabaire inuit, contrat DDC Mission des éditions multimédias, Bibliothèque nationale de France (site Richelieu), co-édition avec la Réunion des musées nationaux, octobre 2001.

2004 Participation au CD-Rom Cultural and Indigenous peoples, oral, written expressions and new technologies, Unesco. Titre de la participation « Les nouveaux contenus de l'écrit et la transmission orale chez les Inuit du Nunavut », co-présentée avec Aaju Peter (Iqaluit, Nunavut). 
